\title{
Diseño de tareas con tecnología: entre investigación y docencia
} Designing digital technology tasks bridging research and mathematics teaching

\author{
A.A. Torres-Rodríguez ${ }^{(D)}$, M. Campos-Nava ${ }^{(D)}$, A. Reyes-Rodríguez ${ }^{(D b}$ b C. Soto-Campos ${ }^{(D)}$ \\ a. Departamento de Ciencias Básicas, Tecnológico Nacional de México campus Atitalaquia, CP 42970 Atitalaquia, Hidalgo, México \\ ${ }^{b}$.Área Académica de Matemáticas y Física, Universidad Autónoma del Estado de Hidalgo, CP 42184, Pachuca, Hidalgo, México
}

\section{Resumen}

En esta contribución, se describe una reflexión derivada del proceso de diseño e implementación de una tarea de aprendizaje con tecnología. Se indica la manera en cómo se incorporaron algunos conceptos e ideas teóricas relacionados con la resolución de problemas, la demanda cognitiva y la mediación instrumental en el diseño de la tarea. Como producto de la reflexión se obtuvo una tabla en la que se describen algunos elementos que se identificaron como esenciales durante el ciclo de diseñoimplementación-rediseño de la tarea.

Palabras Clave: Diseño de tareas, Ley de Grashof, Tecnologías digitales, Resolución de problemas, Reflexión, Matemáticas.

\begin{abstract}
In this paper, we reflect on the process of designing and implementing an instructional task with technology. We explain how some concepts and ideas related to problem-solving, cognitive demand and instrumental mediation were incorporated in the design of the task. As a product of our reflection, we elaborate a table in which we included some elements that were identified as essentials during a design-implementation-redesign cycle.
\end{abstract}

Keywords: Task design, Grashof's law, Digital technologies, Problem solving, Reflection, Mathematics.

\section{Introducción}

De acuerdo con Doyle (1988), las tareas que el profesor implementa en el salón de clase son un elemento clave en la constitución de las características del aprendizaje de los estudiantes. Al abordar ciertas tareas un estudiante llevará a cabo principalmente procedimientos memorísticos o rutinarios, mientras que otras requerirán de un razonamiento conceptual y el establecimiento de conexiones entre diferentes contenidos y procedimientos. Las tareas del primer tipo pueden ofrecer a los estudiantes oportunidades para un aprendizaje memorístico, o para reforzar lo que ya saben; mientras que las segundas favorecen un aprendizaje con entendimiento (Hiebert et al., 1997). ¿Cómo implementar aspectos teóricos de la didáctica de la matemática en el diseño de las tareas? ¿Cómo influyen las tareas diseñadas con base en tales elementos teóricos en las características del conocimiento que los estudiantes construyen al abordarlas?

La perspectiva de resolución de problemas considera que las tareas no rutinarias son el principal recurso con el que cuentan los docentes para favorecer en los estudiantes formas de pensamiento análogas a las que desarrolla un matemático durante su ejercicio profesional (Nieves, Caraballo y Fernández, 2019). Estas tareas se caracterizan porque permiten al estudiante explorar, discriminar entre información relevante de aquella que no lo es, encontrar relaciones entre datos e incógnitas, observar patrones, formular y validar conjeturas, comunicar resultados y elaborar generalizaciones. En palabras de Stein, Remillard y Smith (2007), las tareas que abordan los estudiantes determinan lo que aprenden y cómo lo aprenden.

En este contexto, y dado que una de las funciones principales de los profesores de matemáticas consiste en diseñar tareas de aprendizaje que promuevan entre los estudiantes formas matemáticas de pensar (Schoenfeld, 1992), la presente investigación busca documentar cómo algunas perspectivas teóricas, entre ellas la resolución de problemas, la demanda cognitiva y la mediación instrumental, son de utilidad durante el diseño de una tarea de aprendizaje centrada en desarrollar en los estudiantes un aprendizaje con entendimiento (Hiebert et al., 1997, citado en Barrera-Mora y Reyes-Rodríguez, 2014).

\footnotetext{
*Autor para la correspondencia: agustin.tr@atitalaquia.tecnm.mx Rodríguez, csoto@uaeh.edu.mx (Carlos Soto Campos).

Correo electrónico: agustin.tr@atitalaquia.tecnm.mx (Agustín Alfredo Torres Rodríguez), mcampos@uaeh.edu.mx (Marcos Campos Nava), aaronr@uaeh.edu.mx (Aarón Reyes 


\section{Elementos teóricos considerados para el diseño de la tarea.}

En el diseño de la tarea, consideramos diversos elementos teóricos que a continuación se comparten:

- La perspectiva de resolución de problemas, que aporta elementos didácticos orientados a que los estudiantes desarrollen un aprendizaje basado en el descubrimiento, así como disposición para explorar e investigar relaciones, emplear distintas formas de representación al analizar fenómenos, usar distintos tipos de argumentos y comunicar resultados (Barrera-Mora y Reyes-Rodríguez, 2019). Es decir, que el estudiante use estrategias empleadas comúnmente por los matemáticos al resolver problemas (Santos-Trigo, 2020).

- Con base en la mediación instrumental, el uso de las tecnologías digitales es un elemento que influye en el proceso de aprendizaje, dado su carácter de mediador entre el sujeto que aprende y el objeto de aprendizaje, particularmente centraremos la atención en el efecto del arrastre, en la relación dinámica entre diferentes representaciones, además de las formas en que la tecnología ofrece retroalimentación a las acciones que un estudiante ejecuta al tratar de resolver una situación problemática. Por ejemplo, el uso de una herramienta computacional no sólo hace más potentes algunas heurísticas (estrategias de resolución de problemas), sino que también demanda del estudiante mayores niveles de razonamiento (Barrera-Mora y Reyes-Rodríguez, 2019). Una premisa teórica considerada en este trabajo es que cuando se incluye a las herramientas tecnológicas en el proceso de aprendizaje de las matemáticas, éstas permiten no sólo realizar un mejor trabajo, sino que favorecen formas diferentes de pensar y aprender (Hillmayr, et al., 2020).

En este sentido, Moreno-Armella (2014) afirma que un reto educativo consiste en "diseñar estrategias de articulación de las nuevas tecnologías con las estructuras curriculares actuales" (p.35), sin olvidar que las tecnologías digitales "terminarán por erosionar el currículum y demandarán uno nuevo" (p.35), estrechamente estructurado a partir de sus características.

- El tercer elemento que se incorpora al marco conceptual es el constructo de la demanda cognitiva (Stein y Smith, 1998, citados en Campos y Torres, 2018) de las tareas de aprendizaje matemático, el cual se incluye porque este es un aspecto fundamental para que el estudiante logre el objetivo u objetivos de aprendizaje, esto es, que a través de sus acciones durante la implementación de la tarea, construya conocimiento matemático altamente estructurado. Es importante mencionar que la demanda cognitiva de una tarea matemática puede decaer durante la implementación de esta, por lo cual el profesor debe prestar atención a las acciones de los estudiantes, para promover un ambiente de trabajo en el que el nivel de demanda cognitiva de la tarea se mantenga o incremente. En la tabla 1, se muestran las actividades y procesos asociados con el mantenimiento o disminución de los niveles de demanda cognitiva durante la ejecución de una tarea (Stein y Smith 1998).

Tabla 1: Procesos asociados con el mantenimiento o disminución del nivel de demanda cognitiva. Elaboración propia con base en Stein y Smith (1998).

\begin{tabular}{cc}
\hline Mantenimiento & Disminución \\
\hline $\begin{array}{c}\text { Conducir a los estudiantes } \\
\text { para que proporcionen } \\
\text { justificaciones, } \\
\text { explicaciones y/o }\end{array}$ & $\begin{array}{c}\text { Hacer rutinarios aspectos } \\
\text { problemáticos de la tarea }\end{array}$ \\
significados a través de & \\
preguntas o comentarios. & \\
Seleccionar tareas & Desviar la atención del \\
considerando los & significado, conceptos, o la \\
conocimientos previos del & central de la actividad. \\
estudiante. & Proporcionar poco tiempo \\
Elaborar dibujos con & para entender la tarea, o dar \\
drecuencia, para buscar & los estudiantes queden a la \\
conexiones conceptuales. & deriva. \\
& Seleccionar tareas \\
Proporcionar suficiente & inapropiadas para un grupo \\
tiempo para explorar. & de estudiantes. \\
\hline
\end{tabular}

\section{Metodología}

\subsection{Implementación de los elementos teóricos en el diseño de la tarea.}

Para la implementación, se buscó una tarea en la que el estudiante tuviera que interactuar con objetos matemáticos, y que mediante esta interacción explorara relaciones, realizara observaciones, formulara conjeturas y justificara resultados. En este caso se consideró una actividad dónde no hubiera una ruta predeterminada para obtener la solución del problema, y dónde el estudiante pudiera poner en juego su ingenio, y su creatividad, para desarrollar una o varias rutas de solución.

La tarea que se eligió se enmarca en el contexto de la mecánica, particularmente en el área de la cinemática de mecanismos, en la cual existe un criterio para verificar las condiciones que permiten a una de las barras de un mecanismo de cuatro barras articuladas efectuar revoluciones completas, con relación a alguna de las otras tres. Este criterio se conoce como Ley de Grashof (Shigley, 1970, citado en Reino y Galán, 2020) y tiene relación con las propiedades geométricas de los cuadriláteros. La motivación principal para elegir esta actividad fue que, en la literatura consultada, se enuncia dicho criterio sin hacer una discusión respecto de su validez (Reino y Galán, 2020), con ello se reflexionó que, entonces, dicho criterio podía utilizarse para diseñar una actividad no rutinaria propicia para implementar algunos elementos teóricos relacionados con resolución de problemas y el uso de herramientas digitales, específicamente bajo el precepto de la mediación instrumental.

Se consideró una fase previa a la tarea principal, la cual incluyó una introducción acerca de los mecanismos de 4 barras y su importancia, así como algunos conceptos a manera de antecedentes, como la desigualdad del triángulo; posteriormente se solicitó a los estudiantes emplear una 
herramienta digital para representar el problema y poner en práctica distintas estrategias para solucionarlo.

Debido a ello, el diseño de la actividad consideró los siguientes elementos que desde nuestra perspectiva debe poseer toda tarea: i) Objetivo de aprendizaje o competencias a desarrollar; ii) Elementos matemáticos y competencias previas requeridas; iii) Enunciado y contexto de la actividad; iv) Preparación del escenario y recursos a utilizar; v) Elaboración de posibles rutas hipotéticas de solución y vi) Proceso inquisitivo que permita guiar y extender la actividad. (CamposNava y Torres-Rodríguez, 2017).

Para incluir elementos provenientes de los tres referentes teóricos, se consideraron las siguientes características que debería incluir la tarea: existencia de distintas rutas de solución construibles con el apoyo de la herramienta digital, factibilidad para utilizar casos particulares como medio de avanzar en la solución, posibilidad de plantear hipótesis o conjeturas y la ejecutar estrategias para justificar las conjeturas, entre otras.

Considerando el desarrollo de la actividad, se sugirió el empleo del software CABRI-GÉOMÉTRE II ® (o en lo subsecuente Cabri), es un software de geometría dinámica, diseñado por Jean-Marie Laborde y Franck Bellemain en la Universidad Joseph Fourier de Grenoble, Francia, cuya principal característica, denominada arrastre, dragging en inglés, consiste en la capacidad de un usuario para modificar la posición de ciertos elementos geométricos, como puntos, mediante el mouse, de forma que se mantienen las relaciones estructurales establecidas al crear una construcción geométrica. El sitio oficial del software es https://www.cabri.com/). Se previó que el uso del software permitiría a los estudiantes considerar algunos casos particulares de mecanismos articulados de cuatro barras, cuyo funcionamiento podría visualizarse con apoyo del comando "animación", con lo cual se esperaba que los estudiantes identificaran patrones de comportamiento en las distintas configuraciones geométricas, que plantearan conjeturas sobre el funcionamiento del mecanismo de Grashof, para posteriormente tratar de justificarlas formalmente.

Otro elemento que consideramos relevante para la etapa de implementación de la tarea fue la actividad del profesor, quien debía hacer preguntas que motivaran a los estudiantes para tratar de justificar sus observaciones, comunicar sus ideas y resultados. Este proceso inquisitivo tiene además el objetivo de mantener el nivel de demanda cognitiva de la tarea. Las preguntas que el profesor formulara no debían de ayudar de más al estudiante, pero debían enunciarse de forma tal que guiaran su trabajo y le permitieran encontrar las conexiones propuestas en la ruta hipotética elaborada de forma previa a la implementación de la tarea.

\subsection{Implementación de la tarea con estudiantes.}

La tarea de aprendizaje se implementó con un grupo de doce estudiantes de segundo semestre de una licenciatura en física, en una universidad pública de México. Las edades de los estudiantes estaban comprendidas entre los dieciocho y veinticinco años. Durante el primer semestre de sus estudios de licenciatura, los estudiantes cursaron una asignatura en la cual hicieron uso sistemático de herramientas computacionales para resolver problemas, y en el semestre durante el cual se implementó la tarea, cursaban las asignaturas de Cálculo
Diferencial y Geometría Analítica. La mayoría de los estudiantes manifestó tener poca experiencia con el uso del software de geometría dinámica Cabri, que fue el que se decidió emplear en el diseño de la tarea.

La tarea fue presentada por medio del siguiente enunciado: en mecánica, un mecanismo de cuatro barras no deformables, articuladas en sus extremos, es también conocido como mecanismo de Grashof, si se cumple que al menos una de las barras pueda dar una revolución completa con relación a alguna otra barra. Dado un cuadrilátero cuyas longitudes de sus lados son A, B, C y D, averigüe si es un mecanismo de Grashof. ¿Qué criterio puede usar para saber si un mecanismo de cuatro barras es de Grashof?

Cabe mencionar que, como parte del diseño de la tarea, se inició con una actividad donde se contextualizó la operación de los mecanismos de Grashof a los estudiantes. En el enlace siguiente se encuentran los videos empleados con tal finalidad, https://youtube/eQT9oS2gNVU, los cuales son de libre acceso en la red. Adicionalmente, el docente explicó, algunas aplicaciones de dicho mecanismo, por ejemplo, en los limpiaparabrisas de un automóvil.

\subsection{Una ruta hipotética de solución.}

Simon y Tzur (2004), citados en Inzunza e Islas (2019), mencionan que una trayectoria o ruta hipotética de aprendizaje (THA) se define a través de tres componentes: los objetivos de aprendizaje, la tarea matemática que se empleará para promover dicho aprendizaje y las hipótesis que los estudiantes exploran durante el proceso.

En este orden de ideas, lo que en este trabajo se considera la ruta hipotética de solución que los estudiantes pueden seguir al resolver un problema y que el profesor prevé previamente durante el diseño de la tarea, es compatible con la idea de estos autores. Para diseñar la tarea de aprendizaje se consideró como primer elemento, los conocimientos previos que deben tener los estudiantes que quieran comprender esta tarea para resolverla, ideas básicas de geometría como Ley de senos y cosenos y desigualdad del triángulo, son deseables para tratar de encontrar el criterio solicitado, por otro lado, para entender la tarea, basta con nociones básicas sobre cuadriláteros y movimiento.

Se consideró, asimismo, que la actividad debería tener una primera etapa de preámbulo y contextualización, en la cual primeramente los estudiantes se familiarizaran con la herramienta digital (Cabri) y con el contexto geométrico, por lo que se diseñó un preámbulo, en el cual los estudiantes debían primeramente construir cuadriláteros dadas las longitudes de sus cuatro lados, con lo cual se pretendió que ellos se dieran cuenta que, a diferencia de los triángulos, dadas las longitudes de los lados de un cuadrilátero, este no queda definido, pues los ángulos entre sus lados pueden modificarse conservándose las longitudes, lo que permite introducir el concepto de cuadrilátero articulado.

Posteriormente, dado que el contexto de los mecanismos articulados pudiera no ser cercano a los estudiantes, se diseñó una etapa de contextualización, en la que incluso se proyectó un video breve (buscar como eQT9oS2gNVU en el motor de búsqueda de youtube) donde se muestra el funcionamiento de ese tipo de mecanismos, así como imágenes obtenidas del internet, para aclarar el funcionamiento de un mecanismo 
articulado tipo Grashof (core.ac.uk/download/pdf/11051447, figura 1).

Seguida de esta etapa, se previó que, aunque el enunciado de la actividad quedase claro, los estudiantes pudieran tener dificultades para generar ideas consistentes que les permitieran conjeturar las condiciones del criterio de Grashof, por lo que se diseñaron casos particulares de mecanismos articulados, con los que los estudiantes, podrían llegar a conjeturas plausibles.

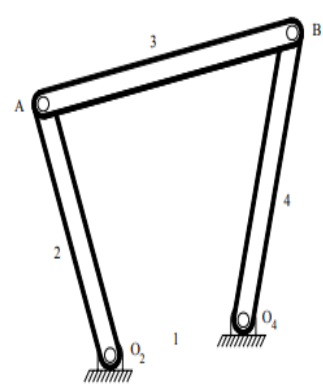

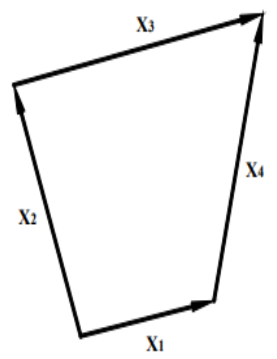

Figura 1. Representaciones de mecanismos de 4 barras.

Posteriormente, la actividad de los estudiantes se enfocó en justificar dichas conjeturas, al considerar que ésta es una actividad importante dentro del enfoque de resolución de problemas, y se relaciona estrechamente con otro elemento que consideramos fundamental: el aprendizaje por descubrimiento. Es importante señalar que en la actividad inicial que fue presencial, se les dio a conocer a los estudiantes algunas ideas sobre los mecanismos de cuatro barras y algunas de sus aplicaciones, además que el docente verificó que los estudiantes no hicieran búsquedas en internet, al menos no antes de la sesión dónde se llevó a cabo la etapa de justificación de conjeturas con el empleo del software. En el diseño de la tarea se consideró que el hecho de que al no explicitar el nombre del criterio permitiría que, a pesar de que los estudiantes realizaran búsquedas en internet, fuese relativamente complicado que los estudiantes localizaran el enunciado de la Ley de Grashof, y de este modo, se garantizaría de cierta manera que durante la etapa de justificación de conjeturas, los estudiantes pudieran arribar mediante sus propios medios a conclusiones similares a las establecidas en dicho criterio.

\section{Resultados de la implementación de la tarea.}

En la fase de análisis se contrastó la ruta hipotética contra la ruta seguida realmente por los estudiantes y se trató de identificar qué variables influyeron en las desviaciones.

A continuación, se muestran los resultados que obtuvieron los estudiantes. Algunos realizaron el análisis de casos particulares, y con ello plantearon conjeturas que intentaron justificar, en tanto que otros estudiantes pusieron su atención en los ángulos que se forman entre las barras del mecanismo, así como en algunos elementos geométricos y trigonométricos. En la figura 2 se muestra un caso particular en el que mecanismo de longitudes 2, 4, 5 y 7 configurado en Cabri puede funcionar como mecanismo de Grashof, al animar la barra más corta (barra A de longitud 2) y mantener inmóvil la barra de longitud 5 , se verifica que puede dar vueltas completas sin problemas.
Este caso particular permitió a los estudiantes conjeturar que si las longitudes de las barras más corta y más larga ( 2 y 7) suman lo mismo que las longitudes de las otras dos barras (4 y 5), entonces una barra del mecanismo puede efectuar giros completos respecto de las otras.

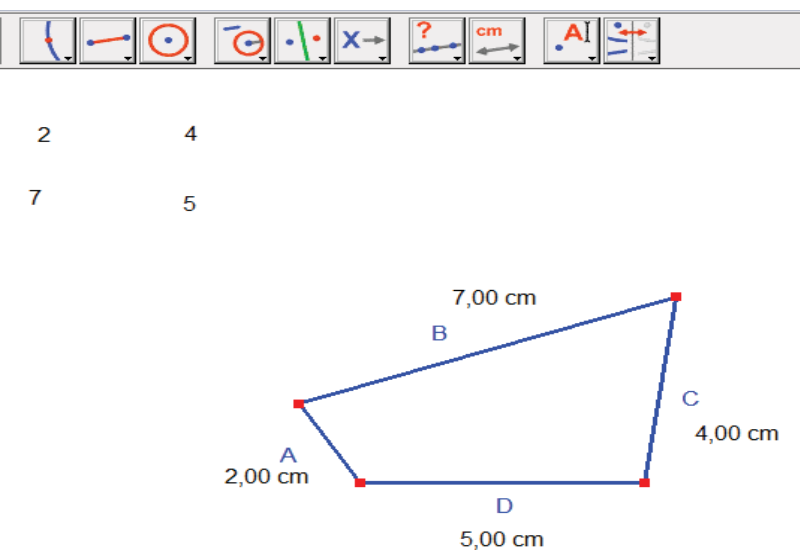

Figura 2: Posible configuración para un mecanismo.

En la figura 3, se muestra otra configuración con cuatro barras de las mismas longitudes que en el caso previo. Al tratar de animar la barra de longitud 4 y mantener estática la barra más larga (longitud 7), se verifica que el mecanismo no puede operar como tipo Grashof.

Este caso (figura 3), puso de relieve que no solo las longitudes de las barras son importantes, sino también el orden en que se construye la configuración, y en este caso particular los estudiantes conjeturaron que la condición de la suma de las longitudes de las barras no era suficiente, si las longitudes de las barras más corta y más larga (2 y 7) suman lo mismo que las longitudes de las otras dos barras ( 4 y 5), el mecanismo no siempre puede operar como tipo Grashof.

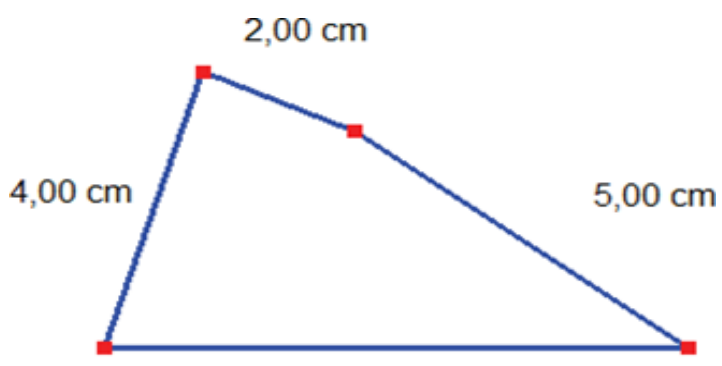

$7,00 \mathrm{~cm}$

Figura 3: Una segunda configuración con las mismas barras.

En la figura. 2 se puede notar que las barras A y C articuladas por uno de sus extremos a la barra D (estática), tratan de efectuar movimientos de rotación pura, sin embargo, la barra B tiene una tendencia muy particular: trata de rotar, pues sus extremos están articulados con los extremos libres de A y C, y además tiene tendencia a trasladarse. Este tipo de movimiento se conoce en cinemática, como movimiento plano general. Los puntos intermedios de la barra B, también llamada barra acopladora, describen por lo general lugares geométricos poco comunes, que suelen representarse con polinomios de hasta sexto grado (Shigley y Uicker, 1999).

Durante la implementación de la tarea se observó que la mayoría de los estudiantes propuso sus propios casos particulares con el objetivo de obtener una mejor comprensión 
del enunciado de la tarea. Propusieron, por ejemplo, el mecanismo de cuatro barras cuyas longitudes fueran 3, 4, 5 y 6 , por otro lado, los estudiantes empezaron a conjeturar que la condición para que la manivela gire completamente tiene que ver con la suma de longitudes dos de las barras y su comparación con la suma de las longitudes de las otras dos.

Otros estudiantes tuvieron la iniciativa de explorar el comportamiento de los ángulos para encontrar relaciones entre las longitudes de los lados, los ángulos y el hecho de que el mecanismo funcione. Un equipo por cuenta propia empezó a cuestionarse ¿qué pasa si en la misma configuración tratamos de dar giros a otra de las barras?, es decir, sugirieron cambiar de manivela. Otro equipo dividió el cuadrilátero en dos triángulos por medio de una diagonal, para analizar los ángulos, lo que les permitió argumentar que las diagonales del mecanismo podían medir a lo más la suma, o a lo menos, la diferencia de dos de las barras. El mismo equipo propuso utilizar la ley de cosenos para tratar de calcular los ángulos del mecanismo en términos de las longitudes de las barras.

Como resultado de la transcripción de la sesión de trabajo, se pudo reunir un conjunto de elementos que pudieron identificarse con varios de los principios que pertenecen a alguno de los 3 referentes teóricos empleados en este trabajo. A continuación, se comparte una tabla (Tabla 2) dónde se resumen algunos de los elementos identificados durante la implementación de la actividad con los estudiantes, así como su clasificación dentro de alguna de las perspectivas teóricas utilizadas como referentes.

Tabla 2: Algunos principios identificados para el diseño de tareas de aprendizaje matemático. Elaborada a partir de los datos colectados durante la implementación.

\begin{tabular}{|c|c|c|}
\hline $\begin{array}{c}\text { Resolución de } \\
\text { problemas }\end{array}$ & Demanda cognitiva & $\begin{array}{c}\text { Mediación } \\
\text { instrumental }\end{array}$ \\
\hline $\begin{array}{l}\text { Las actividades } \\
\text { planteadas deben } \\
\text { centrarse en los } \\
\text { procesos de } \\
\text { pensamiento. }\end{array}$ & $\begin{array}{l}\text { Plantear problemas } \\
\text { que admitan varias } \\
\text { representaciones y } \\
\text { soluciones. }\end{array}$ & $\begin{array}{l}\text { Permitir una } \\
\text { representación } \\
\text { más extensa } \\
\text { que el uso del } \\
\text { lápiz y papel. }\end{array}$ \\
\hline $\begin{array}{l}\text { Favorecer la } \\
\text { autonomía de los } \\
\text { estudiantes. }\end{array}$ & $\begin{array}{l}\text { Crear conexiones } \\
\text { entre distintas áreas } \\
\text { de las matemáticas }\end{array}$ & $\begin{array}{c}\text { Empleo del } \\
\text { software para } \\
\text { contrarrestar } \\
\text { procesos } \\
\text { algorítmicos. }\end{array}$ \\
\hline $\begin{array}{l}\text { Permitir distintas } \\
\text { rutas de solución }\end{array}$ & $\begin{array}{l}\text { Proveer del tiempo } \\
\text { adecuado para la } \\
\text { solución de la tarea. }\end{array}$ & $\begin{array}{l}\text { Favorecer la } \\
\text { identificación } \\
\text { de patrones. }\end{array}$ \\
\hline $\begin{array}{l}\text { Promover y facilitar } \\
\text { la experimentación, la } \\
\text { elaboración de } \\
\text { conjeturas, la } \\
\text { discusión, la } \\
\text { comunicación de } \\
\text { ideas y estrategias. }\end{array}$ & $\begin{array}{c}\text { Que el estudiante } \\
\text { pueda activar } \\
\text { conocimientos } \\
\text { previos. }\end{array}$ & $\begin{array}{l}\text { Identificar y } \\
\text { desarrollar } \\
\text { heurísticas de } \\
\text { solución. }\end{array}$ \\
\hline $\begin{array}{c}\text { Crear conexiones con } \\
\text { conocimientos } \\
\text { previos. }\end{array}$ & $\begin{array}{l}\text { Proceso inquisitivo } \\
\text { que permita la } \\
\text { extensión de la } \\
\text { actividad. }\end{array}$ & $\begin{array}{l}\text { Explorar } \\
\text { diferentes } \\
\text { rutas, y } \\
\text { facilitar la } \\
\text { elaboración y } \\
\text { prueba de } \\
\text { conjeturas. }\end{array}$ \\
\hline
\end{tabular}

\author{
Conectar las \\ matemáticas con otras \\ áreas del \\ conocimiento.
}

Fuente: elaboración propia con los datos obtenidos

\section{Reflexiones finales.}

En términos generales, las observaciones preliminares al concluir la tarea permiten considerar que los objetivos planteados para la misma fueron alcanzados y el software de geometría dinámica fue relevante para tal fin, ya que los estudiantes mostraron disposición para tratar de justificar formalmente sus conjeturas.

Es importante señalar que para poder identificar algunos de los principios mostrados en la tabla 2, los autores revisamos las transcripciones obtenidas, poniendo atención a aquellos estudiantes que evidenciaron algunos de estos principios. Es necesario acotar que aunque el grupo tenía 12 estudiantes, para el desarrollo de la actividad central se organizaron en grupos pequeños, debido a que el interés del profesor siempre fue que los estudiantes fueran realizando sus hallazgos al interactuar con el software, pero también con sus compañeros.

Una de las aportaciones relevantes de este trabajo es que a diferencia de estudios previos relacionados con el diseño de tareas de aprendizaje, se trató de mostrar con detalle cómo se consideraron algunos elementos teóricos para el diseño una tarea en particular, desde la elección de un contexto, el establecimiento los objetivos de aprendizaje, la forma en que los elementos del marco conceptual incidieron en el diseño, que se consideran relevantes para que el profesor propicie un ambiente inquisitivo durante la implementación de la tarea.

Los autores argumentamos que este tipo de resultados pueden servir como base para una fase de rediseño de la tarea y que pueda ensayarse con otros grupos de estudiantes con características similares. Algunas posibles mejoras podrían centrarse en plantear otros casos particulares que promuevan en mayor medida la identificación de patrones, o proporcionar una mejor distribución del tiempo para la prueba de conjeturas. Otro aspecto que puede replantearse podría ser la exposición de resultados por equipos, relacionando este último aspecto con la evaluación de la actividad.

Consideramos, finalmente, que el aprendizaje de diversos tópicos en los cursos de matemáticas o física, pueden abordarse a través de una tarea o actividad de aprendizaje, que incluya el empleo de herramientas digitales que permitan construir conocimiento altamente estructurado, y puedan organizarse en torno a principios derivados de los distintos marcos teóricos y conceptuales que proporcionen los criterios más adecuados para su diseño.

\section{Referencias}

Barrera-Mora, F. y Reyes-Rodriguez, A. (2019). Reasoning processes developed by undergraduate mathematics students during the solution of geometry problem. Far East Journal of Mathematics Education, 19 (1), 61 86.

Barrera-Mora, F. y Reyes-Rodríguez, A. (2014). Sobre el aprendizaje con entendimiento en matemáticas. Revista Pädi, 2(3), DOI:10.29057/icbi.v2i3.525. 
Campos-Nava, M. y Torres-Rodríguez, A. (2018). Diseño de tareas de Aprendizaje matemático con Geogebra: mecanismos articulados. Revista Pädi, 5 (10), 81-86. DOI: https://doi.org/10.29057/icbiv5.i10.

Campos-Nava, M. y Torres-Rodríguez, A. (2017). Las tareas de aprendizaje en la enseñanza de las matemáticas a distancia. Revista Mexicana de Bachillerato a Distancia, 9 (17), 147-155.

Doyle, W. (1988). Work in mathematics classes: the context of students' thinking during instruction. Educational Psychologist, 23, 167-180.

Hiebert, J., Carpenter, T. P., Fennema, E., Fuson, K. C., Wearne, D., Murray, H., Olivier, A. y Human, P. (1997). Making sense: teaching and learning mathematics with understanding. Heinemann, Portsmouth, $\mathrm{NH}$.

Hillmayr, D., Ziernwald, L., Reinhold, F., Hofer, S. y Reiss, K. (2020). The potential of digital tools to enhance mathematics and science learning in secondary schools: A context-specific meta-analysis. Computers and Education, 153, 103897. DOI: 10.1016/j.compedu.2020.103897

Inzunza, S. e Islas, E. (2019). Diseño y Evaluación de una Trayectoria Hipotética de Aprendizaje para intervalos de confianza basada en simulación y datos reales. Revista Bolema, 33 (63), 1-26.

Moreno-Armella, L. (2014). Educación Matemática: del signo al pixel. Ediciones Universidad Distrital Santander, Colombia.

Nieves, S; Caraballo, C.M. y Fernández, C.L. (2019). Metodología para el desarrollo del pensamiento lógico-matemático desde la demostración por inducción complete. Revista Mendive, 17 (3), 393-408.
Reino, M. y Galán, G. (2020), Cinemática de mecanismos Planos, Teoría y Métodos Resueltos. Colección de manuales uex-113. España: Publicaciones de la Universidad de Extremadura.

Santos-Trigo, M. (2020). La Resolución de problemas matemáticos: conectando el trabajo de Polya con el desarrollo de un razonamiento digital. In Yuri Morales-López y Ángel Ruíz (eds) Educación Matemática en las Américas. Comité Interamericano de Educación Matemática, República Dominicana, pp. 29-40.

Schoenfeld, A. H. (1992). Learning to think mathematically: Problem solving, metacognition, and sense making in mathematics. In D. Grouws (ed.) Handbook for Research on Mathematics Teaching and Learning, Macmillan, New York, pp. 334-370.

Stein, M. K., Remillard, J. y Smith, M. (2007). How curriculum influences student learning. In F.K. Lester (ed.) Second handbook of research on mathematics teaching and learning. Macmillan: New York, pp.319-369.

Stein, M. K., y Smith, M. S. (1998). Mathematical tasks as a framework for reflection: from research to practice. Mathematics teaching in the middle school, 3, 268-275. 